\title{
Correlation Between Pharyngeal Airway Space and Sagittal Skeletal Malocclusions
}

\author{
Hina Siddiqui ${ }^{1}$ \\ Sadia Rizwan ${ }^{2}$ \\ Syed Shah Faisal ${ }^{3}$ \\ Syed Sheeraz Hussain ${ }^{4}$
}

\author{
BDS \\ BDS, FCPS \\ BDS, FCPS \\ BDS, DCPS (HCSM), MCPS, FCPS
}

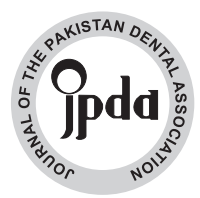

OBJECTIVE: To determine correlation between pharyngeal airway space and sagittal skeletal malocclusions.

METHODOLOGY: Data was collected using pre-treatment records including pre-treatment lateral cephalograms of 54 orthodontic patients. Patients from both gender were included. The age group of selected patients was 15-25 years, Patients belonging to Pakistani ethnicity were included to avoid selection bias. Sagittal skeletal malocclusion was measured by ANB angle to group the skeletal Class I, II and III subjects (ANB $=0-4^{\circ}$, ANB $>4^{\circ}$ and ANB $<0$ respectively). Upper pharyngeal space and Lower Pharyngeal space was measured. This cross sectional study was conducted in Orthodontic OPD at Karachi Medical and Dental College, from August 2019 to March 2020.

RESULTS: The obtained data showed strong negative correlation between ANB angle and upper pharyngeal space whereas moderately negative correlation between ANB angle and lower pharyngeal space.

CONCLUSION: Upper and lower pharyngeal widths were influenced by Sagittal malocclusion.

KEYWORDS: Pharyngeal airway space, Sagittal skeletal malocclusion, Obstructive sleep apnea, Sleep disordered breathing, ANB.

HOW TO CITE: Siddiqui H, Rizwan S, Faisal SS, Hussain SS. Correlation between pharyngeal airway space and sagittal skeletal malocclusions. J Pak Dent Assoc 2020;29(4):217-222.

DOI: https://doi.org/10.25301/JPDA.294.217

Received: 18 August 2020, Accepted: 04 September 2020

\section{INTRODUCTION}

$\mathrm{T}$ he pharyngeal airway space is a structure which contains more than twenty muscles that are committed close to skeletal configurations and there is effect of developing craniofacial skeleton on its dimensions. ${ }^{1}$ It consists of three portions, nasopharynx, oropharynx, and laryngopharynx. ${ }^{2}$ It is a cylindrical structure that covers superoinferiorly from base of cranium to the inferior surface of C6 vertebrae. ${ }^{3}$ The proportions of the superior airway are dictated by arrangements of the craniofacial skeleton, muscles and soft tissue close to the pharynx. ${ }^{4}$ For this void space, effective assessment is necessary. ${ }^{5}$ For the normal development and enlargement

1. FCPS II Trainee, Department of Orthodontics, Karachi Medical and Dental College.

2. Assistant Professor, Department of Orthodontics, Dr. Ishrat-ul-Ibad Khan Institute of Oral Health Sciences.

3. Vice Principal, College of Dentistry, Professor, Department of Orthodontics, Karachi Medical and Dental College.

4. Principal, College of Dentistry, Professor and HOD, Department of Orthodontics, Karachi Medical and Dental College.

Corresponding author: “Dr. Hina Siddiqui” < hinasiddiqui616@gmail.com > of craniofacial region, it necessitates the need of a potent airway. ${ }^{6}$ Genetics is responsible for the dimensions and proportions of face and hence of the air passages; though, environmental conditions seem to be a foremost cause of nasal obstruction. ${ }^{7}$ According to McNamara and Brudon, the upper pharyngeal wall is determined from the posterior surface of the soft palate to the nearest area on the wall of dorsal pharynx, the lower pharyngeal area is determined from the junction of tongue's posterior boundary and the mandible's lower margin to the nearest area on the wall of posterior pharynx. ${ }^{8}$ Narrowing of the pharyngeal airway in the nasopharyngeal region results in breathing through the mouth. ${ }^{9}$ Abnormalities including speech impairment, facial deformities, poor body posture and inadequate teeth positioning are seen in children with mouth breathing. ${ }^{10}$ There is a direct relationship between the mandible position and the airway space has also been established. ${ }^{11}$ A common term used to describe the breathing difficulties during sleep is sleep-disordered breathing (SDB). It is described as chronic increase in airway resistance, partial or full upper airway obstruction which interferes with pulmonary ventilation, oxygenation or sleep quality. ${ }^{12}$ Airway 
constriction in young people can predispose them to obstructive episodes as they mature. ${ }^{13}$ OSA's pathophysiology is sometimes associated with increased upper airway resistance, which might affect the quality of the pulmonary ventilation oxygenation and sleep. ${ }^{14}$ Usual OSA symptoms comprise snoring, sleep apnea and hypopnea, and extreme daytime sleepiness. Left untreated, OSA may also have a strong relationship with greater cardiovascular mortality rates, cognitive impairment, accidents with motor vehicles and lowered quality of life (QOL). ${ }^{15}$ The literature reports various influencing causes for impediment of pharyngeal airways, such as infections, sensitivity reactions and irritating substances in environment. ${ }^{16}$

In several ways malocclusion can be perceived which more likely includes patients with enlarged adenoids, OSAS, snoring and clefts. ${ }^{17}$ The relation between respiratory pattern and form of malocclusion is still disputed. ${ }^{18}$ Patients with craniofacial disorders including a short cranial base, reduction in the cranial base angle, bimaxillary retrusion, and retrognathic mandibles show common finding of narrow airways. ${ }^{19}$ Lateral cephalograms can provide us with useful, credible and replicable airway measurements while minimizing patient costs and radiation exposure. Studies have shown that while cephalometric measurements provide two-dimensional data, cephalometry is a reliable method for airway assessment and adenoid size estimation. ${ }^{20}$

Interpretation of findings in a study conducted by Fadie Khalil showed that group of class II had considerably reduced Upper nasopharyngeal width than group of class I and class III malocclusion ( $\mathrm{p}<0.01)$ i.e.; correlation for class I is -.354 , class II -.422 and for class III 0.157 . McNamara's lower pharyngeal width was statistically larger in group of class III than class I and class II i.e.; correlation for class I is .378, class II .075, and class III -. $118 .^{18}$

So as to predict changes in the upper airway following orthognathic surgeries, with concern of potential development of OSA, it would be helpful to have the data about the upper airway dimensions in Pakistani untreated inhabitants. Hence, the goal of this study is to correlate the pharyngeal dimensions among sagittal skeletal Class I, II, and III orthodontic patients.

\section{METHODOLOGY}

Lateral cephalograms of untreated patients who visited Dental OPD, Karachi Medical and Dental college were recruited in this cross-sectional analytical study. Technique of nonprobability purposive sampling was employed to gather data. Patients from both gender were included. The age group of selected patients was 15-25 years. Patients belonging to Pakistani ethnicity were included to avoid selection bias. Patients who underwent orthodontic treatment, had syndromes, clefts and asymmetric jaws were also excluded. Duration of this study was from August 2019 to March 2020.

All 54 lateral cephalograms signifying subjects in Class I, class II and class III sagittal malocclusion were recruited in the research. Sample size was calculated using correlation sample size calculator, taking statistics for correlation among pharyngeal airway space in upper region and class I as $0.378,95 \%$ confidence interval, the calculated sample size came out as $54 .{ }^{18}$

Data was collected from subjects reporting to the Orthodontics department of Karachi Medical and Dental College after approval from ERC (Ref:- 026/18). All the patients were inspected by researcher. After taking informed consent and thorough history and examination they were enrolled for the study. Measurements were taken on pretreatment lateral cephalograms.

Sagittal skeletal malocclusion: ANB angle to group the sagittal malocclusion in Class I, II and III subjects (ANB from 0 to $4^{\circ}$, ANB greater than $4^{\circ}$ and ANB less than $0^{\circ}$ respectively). ANB angle is formed between Point A (Subspinale), nasion and Point B (supramentale).

McNamara's airways analysis is used to determine pharyngeal airway space which defines:

Upper pharyngeal space: Width of upper pharyngeal area is determined from posterior outline of the soft palate to the nearest point on the wall of posterior pharynx. Its average measurement is 15 to $20 \mathrm{~mm}$.

Lower Pharyngeal space: Width of lower pharyngeal area is measured from juncture of the posterior limit of tongue and the lower boundary of the mandible to the nearby landmark on the wall of posterior pharynx. Its Average value is 10 to $12 \mathrm{~mm}^{8}$

A single investigator who was a fourth year resident in orthodontics manually performed landmark identifications and anatomic tracings. All measurements were recorded on a predesigned Performa.

Data was analyzed using SPSS version 23. Calculations of frequencies and percentages were made for gender, class of malocclusion on the basis of ANB. Mean and SD was calculated for age and space in upper and lower pharyngeal airways. Correlation between space upper and lower regions of pharyngeal airway and skeletal relationship was measured using pearson correlation. Effect modifiers like age and gender were considered through post stratification. Pearson's correlation was applied. P-value lower than or equivalent to 0.05 was taken significant. 


\section{RESULTS}

Descriptive analysis: The sample for our study encompassed 54 number of patients (males $=15$ and females $=39$; having mean age of $17.66 \pm 3.08$ and $19.20 \pm 4.23$ years respectively) comprised of the Class I, class II and class III skeletal malocclusion groups. Patients were grouped according to skeletal pattern into class I $(n=18)$, class II $(\mathrm{n}=19)$ and class III $(\mathrm{n}=17)$. The means and standard deviations of upper and lower regions in pharyngeal airways were calculated.

Table I showed age in relation to space in upper and lower region of pharyngeal airways with Sagittal malocclusion. Mean and standard deviation was noted for 54 number of participants

Table I

\begin{tabular}{|c|c|c|c|}
\hline \multicolumn{4}{|c|}{ Descriptive Statistics } \\
\hline & N & M & SD \\
\hline Age & 54 & 18.7778 & 3.98425 \\
\hline ANB & 54 & 2.8148 & 3.99563 \\
\hline UPS & 54 & 13.3519 & 2.34848 \\
\hline LPS & 54 & 11.8519 & 3.87862 \\
\hline
\end{tabular}

Inferential analysis: SPSS version 23 was used to analyze data. Calculations of Frequency and percentages were made for gender, class of malocclusion on the basis of ANB. Mean and SD was calculated for age and upper and lower pharyngeal airway space. Correlation between space in regions of upper and lower pharyngeal airways and skeletal relationship was measured using Pearson product moment correlation. Effect modifiers like age and gender were considered through post stratification. Pearson product moment correlation was applied; P-value was kept lower than or equal to 0.05 to be significant.

McNamara's upper pharyngeal space: Results showed that mean values are smaller for group of class II than class I and class III malocclusion whereas group of class III malocclusion showed larger space in upper pharynx than class I and class II.

McNamara's lower pharyngeal space: Results showed that mean values are smaller for class II group than class I and class III and class III showed larger upper pharyngeal space than class I and class II.

Pearson product moment correlation showed strong negative correlation exists between ANB angle and upper pharyngeal space which was statistically significant at 0.01 level and moderately negative correlation exists between ANB angle and lower pharyngeal space which was statistically significant at 0.05 level.

One-way ANOVA was applied for comparison of upper and lower airways between groups and we found statistically significant difference class III skeletal group when compared with class II and class I skeletal malocclusion.

In Table II it is shown that there is a significant negative moderate correlation between upper pharyngeal space and Sagittal skeletal malocclusion mean value is -0.530 . There

Table II

\begin{tabular}{cccc}
\hline \multicolumn{4}{c}{ Correlations } \\
\hline & ANB & UPS & LPS \\
\hline ANB & - & $-.530^{* *}$ & $-.345^{*}$ \\
UPS & & - & $\mathbf{. 5 2 2}^{* *}$ \\
LPS & & & - \\
\hline
\end{tabular}

**. Correlation is significant at the 0.01 level (2-tailed).

*. Correlation is significant at the 0.05 level (2-tailed).

is significant negative weak correlation between lower pharyngeal space and Sagittal skeletal malocclusion mean value is -0.345 .

Table III showed the result for class 1, class II and class III comparison with pharyngeal airway space. One-way ANOVA was applied to see the mean difference. The result is significant for the class I, II and III mean difference compared with space in regions of upper and lower pharynx.

Table III

\begin{tabular}{|c|c|c|c|c|c|}
\hline & N & Class I & Class II & Class III & P-value \\
\hline ANB & 18 & $03.06(1.06)$ & $06.63(3.25)$ & $-1.71(0.85)$ & $<.001$ \\
\hline UPS & 19 & $13.17(2.33)$ & $11.95(1.75)$ & $15.12(1.83)$ & $<.001$ \\
\hline LPS & 17 & $11.72(4.47)$ & $10.45(2.49)$ & $13.56(4.02)$ & .052 \\
\hline
\end{tabular}

The highest mean difference was noted for class III in regions of upper and lower pharynx having mean values 15.12 and 13.56 was noted respectively. The highest mean difference for sagittal skeletal malocclusion was noted in class II skeletal malocclusion that is 06.63 .

\section{DISCUSSION}

The present study aimed to confirm hypothesis if there is any correlation exists between pharyngeal airway space and sagittal skeletal relationships in Pakistani population. 
The obtained data showed strong negative correlation exists between ANB angle and upper pharyngeal space and moderately negative correlation exists between ANB angle and lower pharyngeal space. ANB angle is taken to classify sagittal skeletal malocclusion.

Our findings revealed while increasing ANB angle as in skeletal class II cases, there is a decrease in space in regions of upper and lower pharynx and in decreased ANB cases i.e.; in skeletal class III cases space in regions of upper and lower pharynx is increased. The obtained outcomes also found statistically significant difference for space in upper airways for skeletal class III group when compared with skeletal class II and skeletal class I.

The findings of this study revealed statistically significant mean differences among class I, II and III cases, showing narrow space of airways in cases of class II and wider in cases of class III. The highest mean difference was noted for class III in spaces of upper and lower pharyngeal airways having mean values of 5.12 and 13.56 was noted respectively. Ceylan and Oktay; in their study showed variations in the angle of ANB which affected size of nasopharyngeal airway, and that the space of oropharyngeal area was decreased in patients having greater ANB angle, these findings are in favor with our study. ${ }^{21}$ Moto et al. evaluated the diameter of the pharyngeal airway at with a normal, retruded and protruded mandible. Their results showed largest Pharyngeal airway space in the group having prognathic mandible trailed by the groups of normal mandible and retrognathic mandible. These findings showed that the measurement of the pharyngeal airway is influenced by different mandibular skeletal positions which is in favor with our findings. ${ }^{22}$ Findings obtained by Kerr et al., showed patients having Class II skeletal malocclusion had reduced nasopharyngeal and adenoid areas when compared with class I. ${ }^{23}$ A study by Atia Abd et al. also showed narrow dimensions of airways in Class II division 1 malocclusion patients when differentiated with Class I malocclusion and Class III malocclusion, whereas Class III malocclusion had wider dimensions of lower pharynx because of prognathic mandible, these results are also consistent with our results. ${ }^{24}$

Sarwat Memon et al, compared craniofacial forms with spaces in pharyngeal airways in 360 orthodontic patients. They reported that upper pharyngeal space is not influenced by skeletal malocclusion. ${ }^{16}$ These results contradict with our study which showed a significant correlation exists of airway space in upper and lower pharynx with ANB which represent sagittal malocclusion. Class II patients have narrower airway width in upper and lower regions of pharynx as compared to class III and I, and class III has wider space in upper and lower pharynx compared to class I and class II. Though, in our study vertical growth was not highlighted.
In a study conducted by Freitas et al; his findings showed vertical growers have significantly reduced upper pharyngeal airways whereas, there was no influence of the type of malocclusion with upper and lower width of pharyngeal airways. ${ }^{25}$ Their findings contradict the findings of this study which exhibited significantly moderate correlation in upper pharyngeal space and significant weak correlation in lower airway space with sagittal malocclusion. In a study conducted by Sosa et al., while relationship between adenoids and malocclusion was analyzed, he concluded that airways dimensions did not change while changing malocclusion type. They showed lower correlations among the dimensions of the area of nasopharynx and sagittal malocclusion, whereas our research showed strong correlations of skeletal malocclusion with upper pharyngeal airway space and moderate correlations of skeletal malocclusion with lower pharyngeal space. ${ }^{26}$ Our outcomes concerning the pharyngeal space and anteroposterior skeletal malocclusion relationship are not consistent with studies that showed no relationship between pharyngeal space and sagittal malocclusion. Nonetheless, different variables were utilized in previous studies, which made it challenging to compare. Batool et al. described that individuals having Class II malocclusion and growth in vertical direction have significantly narrow airways than Class II malocclusion and horizontal growing pattern. ${ }^{7}$

Sagittal class II malocclusions are categorized by retrognathic mandible, prognathic maxilla, or a blend of both. To correct skeletal class II malocclusion, three interventions linked with growth are utilized: modification of growth, camouflage, or surgery. ${ }^{27}$ While planning treatment for patients requiring orthognathic surgery, maxillary setback can further reduce upper airway dimensions, for this purpose it is necessary to determine the effect of anteroposterior positioning of jaw bones.

\section{LIMITATIONS}

It is necessary to mention two study limitations:

1. Two-dimensional images are considered for evaluation of airway which is a 3-D structure.

2. Vertical pattern of growth was not emphasized which influence the rotation of mandible. Further emphasis on vertical pattern is also needed.

\section{CONCLUSIONS}

Considering the outcomes obtained in our study, we conclude that:

1. Anteroposterior relationship of maxilla and mandible is correlated with pharyngeal airway space dimensions. 
2. Skeletal class III malocclusion has increased dimension of airways in upper and lower regions and class II malocclusion has reduced dimensions of upper and lower region of airways.

\section{CONFLICT OF INTEREST}

None declared.

\section{FUNDING DISCLOSURE}

No funds availed from any organization or institute.

\section{REFERENCES}

1. Kumar A, Nandi MK. Correlation between Pharyngeal Airway Space and Craniofacial Morphology-A Cephalometric Study. (IOSRJDMS). 2017;16:43-7 https://doi.org/10.9790/0853-1603104347

2. Chen CM, Lai S, Chen KK, Lee HE. Correlation between the pharyngeal airway space and head posture after surgery for mandibular prognathism. BioMed Res. Int. 2015;Article ID 251021.

https://doi.org/10.1155/2015/251021

3. Babu ZK, Senthilkumar T, Kumar S, Dogra S, Sharma A, Sharma $\mathrm{N}$, et al. Comparison of Uvulo-glossopharyngeal Dimensions between Hypo-and Hyper-divergent Growth Pattern of Skeletal Class II Malocclusion in Local Population: A Pilot Study. J Indian Acad Dent Spec Res 2017;4:1-4

https://doi.org/10.4103/jiadsr.jiadsr_15_17

4. Shokri A, Miresmaeili A, Ahmadi A, Amini P, Falah-Kooshki S. Comparison of pharyngeal airway volume in different skeletal facial patterns using cone beam computed tomography. J Clin Exp Dent. 2018;10:e1017.

https://doi.org/10.4317/jced.55033

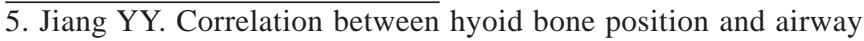
dimensions in Chinese adolescents by cone beam computed tomography analysis. Int J Oral Maxillofac Surg. 2016;45:914-21.

https://doi.org/10.1016/j.ijom.2016.02.005

6. Soni J, Shyagali TR, Bhayya DP, Shah R. Evaluation of pharyngeal space in different combinations of Class II skeletal malocclusion. Acta Informatica Medica. 2015;23:285.

https://doi.org/10.5455/aim.2015.23.285-289

7. Batool I, Shaheed M, Rizvi SAA, Assad A. Comparison of upper and lower pharyngeal airway space in class II high and low angle cases. Pak. oral dent. J. 2010;30-81.

8. McNamara JA, Brudon WL. Orthodontic and orthopedic treatment in the mixed dentition. Needham Press; 1993.

9. Lopatiene K, Šidlauskas A, Vasiliauskas A, Cecyte L, Švalkauskiene V, Šidlauskas M. Relationship between malocclusion, soft tissue profile, and pharyngeal airways: A cephalometric study. Medicina. 2016;52:307-14.

https://doi.org/10.1016/j.medici.2016.09.005

10. Grippaudo C, Paolantonio EG, Antonini G, Saulle R, La Torre G, Deli R. Association between oral habits, mouth breathing and malocclusion. Acta Otorhinolaryngologica Italica. 2016;36:386-94.

11. Jiang C, Yi Y, Jiang C, Fang S, Wang J. Pharyngeal airway space and hyoid bone positioning after different orthognathic surgeries in skeletal Class II patients. J. Oral Maxillofac. Surg. 2017;75:1482-90. https://doi.org/10.1016/j.joms.2017.02.021

12. Pavoni C, Lombardo EC, Lione R, Bollero P, Ottaviani F, Cozza P. Orthopaedic treatment effects of functional therapy on the sagittal pharyngeal dimensions in subjects with sleep-disordered breathing and Class II malocclusion. Acta Otorhinolaryngologica Italica. 2017;37:479-85.

13. Ansar J, Singh RK, Bhattacharya P, Agarwal DK, Verma SK, Maheshwari S. Cephalometric evaluation of the airway dimensions in subjects with different growth patterns. J. Orthod. Res. 2015;3:108. https://doi.org/10.4103/2321-3825.149051

14. Pisacane S, Carotenuto M, d'Apuzzo F, Vitale M, Grassia V, FloresMir C, et al. Cephalometric evaluation of craniofacial morphology in pediatric patients with fully diagnosed OSA with distinct sagittal skeletal malocclusions. J Dent Sleep Med. 2019;6(4)

https://doi.org/10.15331/jdsm.7096

15. Cui DM, Han DM, Nicolas B, Hu CL, Wu J, Su MM. Threedimensional evaluation of nasal surgery in patients with obstructive sleep apnea. Chin. Med. J. 2016;129:651.

https://doi.org/10.4103/0366-6999.177971

16. Memon S, Fida M, Shaikh A. Comparison of different craniofacial patterns with pharyngeal widths. J Coll Physicians Surg Pak. 2012;22:302.

17. Bhambri E, Ahuja V, Ahuja S, Bhambri G, Choudhary A, Sukhija $\mathrm{S}$. The association between soft palate shape and Need's ratio in various sagittal skeletal malocclusions: A digital lateral cephalometric study. Int. J. Orthod. Rehabil. 2018;9:8.

https://doi.org/10.4103/ijor.ijor_41_17

18. Saeed G.et al, Comparative study of airways in the saggital plane with mcnamara analysis on lateral cephalometric analysis, Int J Dent Health Sci 2016; 3:868-875

19. Ize-Iyamu IN. Pharyngeal dimensions in skeletal class I, II, and III orthodontic patients in a Nigerian population. West African J Radiol. 2016;23:89-94.

https://doi.org/10.4103/1115-3474.187967

20. Pirilä-Parkkinen K, Löppönen H, Nieminen P, Tolonen U, Pääkkö E, Pirttiniemi P. Validity of upper airway assessment in children: a clinical, cephalometric, and MRI study. Angle Orthod. 2011;813:4339.

https://doi.org/10.2319/063010-362.1 
21. Ceylan I, Oktay H. A study on the pharyngeal size in different skeletal patterns. Am J Orthod Dentofacial Orthop. 1995;108:69-75. https://doi.org/10.1016/S0889-5406(95)70068-4

22. Muto T, Yamazaki A, Takeda S. A cephalometric evaluation of the pharyngeal airway space in patients with mandibular retrognathia and prognathia, and normal subjects. Int J Oral Maxillofac Surg. 2008;37:228-31.

https://doi.org/10.1016/j.ijom.2007.06.020

23. Kerr WJ. The nasopharynx, face height, and overbite. Angle Orthod. 1985;55:31-6.

24. Yousif AA. Evaluation of upper and lower pharyngeal airway in hypo and hyper divergent Class I, II and III malocclusions in a group of Egyptian patients. Tanta Dent. J. 2015;12:265-76.

https://doi.org/10.1016/j.tdj.2015.07.001
25. de Freitas MR, Alcazar NM, Janson G, de Freitas KM, Henriques JF. Upper and lower pharyngeal airways in subjects with Class I and Class II malocclusions and different growth patterns. Am J Orthod Dentofacial Orthop. 2006;130:742-5.

https://doi.org/10.1016/j.ajodo.2005.01.033

26. Sosa FA, Graber TM, Muller TP. Postpharyngeal lymphoid tissue in Angle Class I and Class II malocclusions. Am. J. Orthod. 1982;81:299309.

https://doi.org/10.1016/0002-9416(82)90216-0

27. Uslu-Akcam O. Pharyngeal airway dimensions in skeletal class II: A cephalometric growth study. Imaging Sci Dent. 2017;47:1-9. https://doi.org/10.5624/isd.2017.47.1.1 